\title{
Spontaneous Bilateral Broad Ligament Rupture Associated with a Connective Tissue Disorder
}

\author{
Jacques-Turcotte L MD ${ }^{1}$, Mahone M MD ${ }^{1,2}$, Reichetzer B MD ${ }^{3}$, Elhaffaf $\mathrm{Z} \mathrm{MD}^{4}$ and Weber F $\mathrm{MD}^{1 *}$ \\ ${ }^{1}$ Internal Medicine Division, Department of Medicine, Centre Hospitalier de l'Université de Montréal (CHUM), Montreal, Canada \\ ${ }^{2}$ Centre de recherche CHUM, Montreal, Canada \\ ${ }^{3}$ Department Obstetrics and Gynecology, Centre Hospitalier de l'Université de Montréal (CHUM), Montréal, Canada \\ ${ }^{4}$ Genetic Division, Departement of Medicine, Centre Hospitalier de l'Université de Montréal (CHUM), Canada
}

Submission: August 16, 2017 ; Published: August 28, 2017

*Corresponding author: Florence Weber, Internal Medicine Division, Department of Medicine, Centre Hospitalier de l Universit de Montr al (CHUM), 1058 Saint-Denis Street, Montreal, QC, Canada, Fax: 514-890-8000; Email: florence.weber@umontreal.ca

\begin{abstract}
Background: Hemoperitoneum secondary to spontaneous rupture of uterine vessels in the broad ligament during pregnancy usually results from trauma or varicose vein dilatation, leading to uterine vessel rupture.

Case: A 33-year-old primigravida presented to the hospital for abdominal pain. Fetal distress led to an urgent caesarean. Abdominal exploration revealed hemoperitoneum secondary to bilateral lesion of the broad ligament and abnormal hysterotomia extension. The patient was referred for genetic evaluation of connective tissue disease. A fibrillin-1 (FBN-1) mutation was discovered; a mutation associated with Marfan syndrome.
\end{abstract}

Conclusion: Although this patient had no other signs suggesting Marfan syndrome, the FBN-1 mutation may explain the bilateral broad ligament rupture without trauma. We recommend screening for connective tissue disease in patients with this complication.

Keywords: Broad ligament rupture; Marfan; Hemoperitoneum; Uterine vein rupture, Pregnancy

\section{Introduction}

There have been a few reported cases of hemoperitoneum following rupture of uterine vessels or rupture of broad ligament in pregnant women [1]. The classic presentation is acute abdominal pain with peritonitism, often accompanied by maternal shock and fetal distress. The high probability of maternal and fetal morbidity and mortality warrants urgent surgical management. A spontaneous rupture of uterine vessels is identified in the majority of hemoperitoneum cases, and 75\% of these are located in the broad ligament [2]. Uterine vessel ruptures are most likely caused by a preceding trauma within $24 \mathrm{~h}$, or linked to varicose dilation of uterine veins. To date, only 4 cases of spontaneous rupture of the broad ligament during pregnancy without varicose veins have been reported. Two of these cases report modest trauma the same day as the hemoperitoneum (horse carriage riding and deceleration in a car) $[3,4]$. We report a case of hemoperitoneum secondary to spontaneous bilateral broad ligament rupture at 35 weeks of gestation, without either trauma or uterine vessel varicosity. The patient was found to have a mutation in the fibrillin-1 (FNB-1) gene.

\section{Case}

A 33-year-old primigravida, originally from Cameroon, presented 3 times to the hospital over 2 weeks in 2015 for abdominal pain and irregular contractions at around 33 weeks of gestation. She was known only for pre-gestational hypothyroidism, but had developed gestational diabetes. Initially, cystitis with uterine irritability was suspected and treated with amoxicillin. The patient returned a few days later, at 34+5 weeks for progressive abdominal pain. Fetal evaluation was reassuring and vaginal examination showed a closed cervix. She was sent home with a follow-up appointment. However, due to substantial abdominal pain, regular contractions and a decrease in fetal movements, the patient presented to the delivery room again 4 days later. The patient $\mathrm{s}$ vital signs were normal except for sinus tachycardia at 102 beats per minute (bpm).

She went into spontaneous labour. The fetus developed variable and late decelerations followed by bradycardia at $70 \mathrm{bpm}$ lasting 4 minutes. An emergency caesarean section was performed via lower uterine transverse incision. A live 
female baby was delivery without complication. The baby weighed 2315g, with Apgar scores of 6, 8 and 9 at 1, at 5 and 10 minutes respectively. The umbilical arterial $\mathrm{pH}$ was 7.26. A hemoperitoneum of $700 \mathrm{ml}$ with clots was discovered on entry into the abdominal cavity. An abdominal exploration revealed bilateral lesions of the broad ligaments, an abnormal bilateral extension of the hysterotomia to the posterior face of the uterus to the uterosacral ligaments and a $3 \mathrm{~cm}$ lesion on the bladder serosa. There was no evidence of varicose veins. The gynaecologist performed a double-layer closure of the uterus, followed by a repair of both broad ligaments and of the bladder after insuring there was no leak of methylene blue. The total blood loss was estimated to be 1.5 litres. The patient was given 2 blood transfusions and remained stable. Her evolution after the surgery was unremarkable other than a paralytic ileus.

Following this dramatic event, the patient was investigated for possible connective tissue disorder. She reported no joint laxity, ocular abnormalities or striae atrophicae which are all associated with connective tissue disorders such as Marfan syndrome and Ehlers Danlos. The patient had previously had skin graft surgery for a burn on the thigh which healed normally. The patient, originally from non-consanguineous Cameroonian family, was then referred for genetic evaluation. Little information was available about the patient's s family history. Genetic screening revealed a fibrillin-1 mutation (FNB-1; NM_000138.4:c.7867C>T (p.His2623Tyr)) that may be associated with Marfan syndrome. However, there was no other clinical element suggestive of this syndrome. Additionally, a mutation in ZNF469 was also identified, but was of uncertain significance and may have had no clinical impact.

The patient subsequently developed type 2 diabetes. When she subsequently became pregnant again, a follow-up with cardiac ultrasound was performed at 33 weeks of gestation due to the FNB-1 mutation. A normal aortic root $(2.8 \mathrm{~cm})$ was noted. In the third trimester the patient suffered an episode of vaginal bleeding secondary to abruptio placentae.

In view of the FNB-1 mutation and the complications of the first pregnancy, a multidisciplinary team of gynaecologists, obstetrical medicine specialists and geneticists decided that elective caesarean section be performed at 36 weeks of gestation. The surgery was performed under spinal anaesthesia and a live male baby weighing $2470 \mathrm{~g}$ was delivered. The broad ligaments were visualized and there was a fenestration in the left broad ligament; the right broad ligament was torn but intact.

\section{Discussion}

Hemoperitoneum secondary to uterine vessel rupture during pregnancy or following delivery is a rare occurrence. Most of these are in the context of uterine varicose veins or minor trauma leading to a sudden anteroposterior movement of a gravid uterus [4]. Many authors suspect that there may be a physiological component to the vascular rupture of uterine vessels [5]. The risk of rupture may be augmented by the increase in venous pressure in the utero-ovarian vessels during pregnancy due to hormonal and mechanical changes, the absence of valves in these vessels, the weakness of the vessel walls caused by atrophy of muscular layer and the free anatomises of the utero-ovarian vessels in the broad ligament [6]. Moreover, arteriovenous malformation and local inflammation secondary to endometriosis may also promote this complication[6-8]. In this context, minor trauma, cough, defecation or uterine contractions, may contribute to the rupture of these weakened uterine vessels. No link with age or parity was reported in the literature and there were no recommendations concerning subsequent pregnancies [9].

Venous insufficiency is suspected to contribute to this phenomenon. Some authors have suggested applying the same recommendations as with lower limb venous insufficiency: regular physical activity, reducing excess weight, elevation of the legs, lying in left lateral position, and avoiding prolonged standing. Color Doppler echography has also been proposed to document uterine varicosity recurrence. Efficient monitoring and effective treatment methods following the diagnosis of uterine varicosity remains unclear [10].

Another case of spontaneous broad ligament tear in a primigravida during the beginning of labour, without a history of trauma and without uterine varicosity, was reported in the United Kingdom [11]. Two other cases of spontaneous broad ligament rupture were published in France, but in these cases, uterine varicosities were objective during the surgery, without other signs of venous insufficiency [7-10].

We publish the first case of spontaneous broad ligament bilateral rupture during pregnancy possibly associated with a mutation present in connective tissue disease; the Marfan syndrome. Marfan syndrome is a systemic disorder caused by a mutation in the gene encoding fibrillin 1 (FBN-1) and transmitted as an autosomal dominant trait. This syndrome may affect cardiovascular, skeletal and ocular systems. The diagnosis is based on the Ghent criteria, which include family history [12].

The major issue concerning Marfan syndrome and pregnancy is the risk of cardiovascular complication such as aortic dissection, dilatation of aortic root, mitral valve prolapsed and dilatation of the pulmonary artery [12-13]. Furthermore, lumbosacral dural ectasia presents in $70 \%$ patients with Marfan syndrome and necessitates an anaesthesiology evaluation before epidural [12]. Few cases have been reported of postpartum hemorrhage in Marfan syndrome. Some authors suspect that degenerative vascular change may be present in the uterine vessels and that pregnancy may precipitate or exacerbate dilatation and dissection of already diseased vessels [14]. Recently, one case of postpartum hemorrhage was reported in a woman with Marfan syndrome presenting numerous dilated and tortuous blood vessels on the inner uterine aspect. During histopathological analysis, fragmentation of the elastic lamina of the dilated vessels and mucoid degeneration of the walls was noted. In this case, the postpartum hemorrhage was attributed to vascular modification secondary to the Marfan syndrome [14]. 
The patient presented here had the mutation FNB-1 NM_000138.4:c.7867C >T (p.His2623Tyr). This mutation is located in the EGF-like calcium-binding protein. A small modification may change the polarity of the protein and alter the function [15]. A vascular follow-up was recommended for the patient and her children because of these mutations. Connective tissue disorders and their genetic mutations are principally described in the Caucasian population. No data is available to confirm that this presentation and the described genetic mutations are the same in other ethnicities; some patients may have different clinical presentations associated with connective tissue disorder.

\section{Conclusion}

An FNB-1 mutation was discovered in this patient, but no clinical element supported a connective tissue's disorder except for the abnormal extension of the hysterotomia and the spontaneous bilateral rupture of broad ligament during her first pregnancy. At this time, based on the Ghent criteria, a diagnosis of Marfan syndrome cannot be given. Otherwise, in the context of the idiopathic obstetrical complication, we suspect that the presence of FNB-1 mutation may have played a role in the tissue $\mathrm{s}$ excessive fragility. Based on this case, we recommend that genetic screening for connective tissue diseases should be done in patients with spontaneous broad ligament rupture, despite the lack of information available about these genetic tests for different ethnicities.

\section{Acknowledgements}

The authors thank Mr. John Davison for editing the manuscript.

\section{References}

1. LaRose P, Sehdeva PK (1978) Spontaneous rupture of a uterine vein during labor. South Med J 719110: 1446-1447.

2. Ziereisen V, Bellens B, Gérard C, Baeyens L (2003) Spontaneous rupture of utero-ovarian vessels in postpartal period: a case report and review of the literature. J Gynecol obstet Biol Reprod 32(1): 51-54.
3. Chowdhury RR, Ahern T, Mckenzie GB (2004) Prelabour rupture of the broad ligament in a primigravida. BJOG 111(2): 181.

4. Descheemaeker V, Morcel K, Aguillela DC, Le Nouvel JB, et al. (2006) Une cause rare de douleur pelvienne aiguë au troisième trimestre de grossesse: la rupture des ligaments larges. Douleurs 7(2): 75-77.

5. Aissi G, Gaudineau A, Trieu NT, Sananes N, Boisramé T, et al. (2014) Hémopéritoine exceptionnel du troisième trimestre de la grossesse. Gynécologie Obstétrique \& Fertilité 42: 441-443.

6. Micheletti G (1969) Massive hemoperitoneum caused by rupture of uterine varices at the early stage of labor in normal pregnancy, followed by shock and heart arrest. Minerva Ginecol 21(10): 655-658.

7. Inoue T, Moriwaki T, Niki I (1992) Endometriosis and spontaneous rupture of utero-ovarian vessels during pregnancy. Lancet 340(8813): 240-241.

8. Huisman CMA, Boers KE (2010) Spontaneous rupture of broad ligament and uterine vessels during pregnancy. Acta Obstet Gynecol Scand 89(10): 1368-1369.

9. Bellucci MJ, Burke MC, Querusio L (1994) Atraumatic rupture of uteroovarian vessels during pregnancy: a lethal presentation of maternal shock. Ann Emerg Med 23(2): 360-362.

10. Roger N, Chitrit Y, Souhaid A, Rezig K, Saint LS (2005) Hémopéritoine par rupture de varices utérines en cours de grossesse. J Gynecol Obstet Biol Reprod 34(5): 497-500.

11. Soleymanimajd H, Datta S, Iqbal R (2007) Spontaneous broad ligament tear in early labour. J Obstet Gynaecol 27(6): 627-628.

12. Carboni S, Capucci R, Pivato E, Poggi A, Patella A (2013) Marfan's syndrome and pregnancy: a good maternal and fetal outcome. J Prenat Med 7(2): 21-24.

13. Elkayam U, Ostrzega E, Shotan A Mehra A (1995) Cardiovascular Problems in Pregnant Women with the Marfan syndrome. Ann Intern Med 123(2): 117-122.

14. Irons DW, Pollard KP (1993) Post partum haemorrhage secondary to Marfan's disease of the uterine vasculature. Br J Obstet Gynaecol 100(3): 279-281.

15. Collod BG, Béroud C, Adès L, Black C, Boxer M et al. (1997) Marfan Database (second edition): software and database for the analysis of mutations in the human FBN1 gene. Nucleic Acids Research 25(1): 147-150.

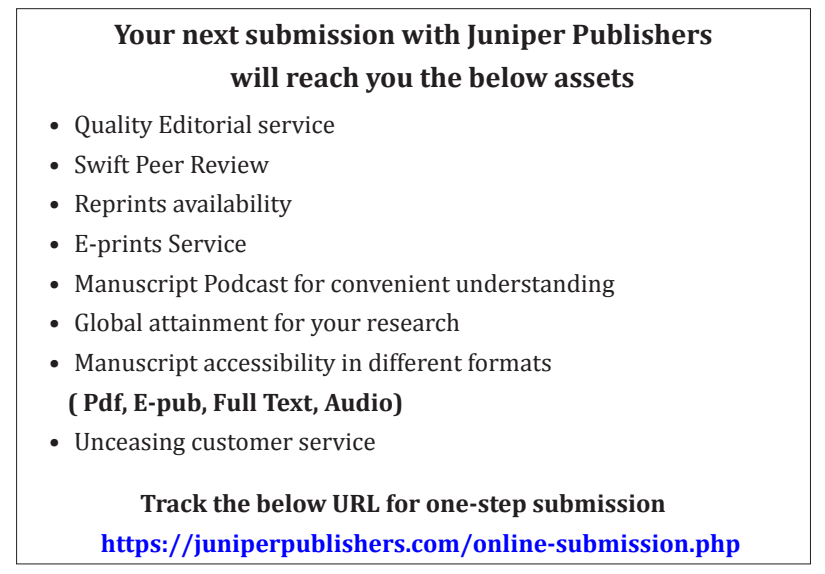

